\title{
Papers
}

\section{Randomised trial of cranberry-lingonberry juice and Lactobacillus GG drink for the prevention of urinary tract infections in women}

Tero Kontiokari, Kaj Sundqvist, M Nuutinen, T Pokka, M Koskela, M Uhari

\begin{abstract}
Objective To determine whether recurrences of urinary tract infection can be prevented with cranberry-lingonberry juice or with Lactobacillus GG drink.

Design Open, randomised controlled 12 month follow up trial.

Setting Health centres for university students and staff of university hospital.

Participants 150 women with urinary tract infection caused by Escherichia coli randomly allocated into three groups.

Interventions $50 \mathrm{ml}$ of cranberry-lingonberry juice concentrate daily for six months or $100 \mathrm{ml}$ of lactobacillus drink five days a week for one year, or no intervention.

Main outcome measure First recurrence of symptomatic urinary tract infection, defined as bacterial growth $\geqslant 10^{5}$ colony forming units $/ \mathrm{ml}$ in a clean voided midstream urine specimen.

Results The cumulative rate of first recurrence of urinary tract infection during the 12 month follow up differed significantly between the groups $(\mathrm{P}=0.048)$. At six months, eight (16\%) women in the cranberry group, 19 (39\%) in the lactobacillus group, and 18 $(36 \%)$ in the control group had had at least one recurrence. This is a $20 \%$ reduction in absolute risk in the cranberry group compared with the control group (95\% confidence interval $3 \%$ to $36 \%, \mathrm{P}=0.023$, number needed to treat $=5,95 \%$ confidence interval 3 to 34$)$.

Conclusion Regular drinking of cranberry juice but not lactobacillus seems to reduce the recurrence of urinary tract infection.
\end{abstract}

\section{Introduction}

Up to $60 \%$ of women have a urinary tract infection at some point in their life. ${ }^{1}$ Sexual activity is the most important risk factor for urinary tract infection. ${ }^{2}$ At least a third of women with urinary tract infection will experience a recurrence during the following year, with recurrence being most common in the age groups 25-9 and over 55 years. ${ }^{3}$ Recurrences are a common indication for long term antimicrobial prophylaxis, but emerging antimicrobial resistance underlines the need for alternatives. ${ }^{45}$

The bacteria causing urinary tract infection arise from the stools. Dietary changes can alter the balance of faecal bacteria. ${ }^{6}$ Vaccinium berries and products containing lactobacilli have been shown to act against the coliform bacteria that cause most urinary tract infections. ${ }^{78}$ Cranberry juice prevents bacteriuria in elderly women, ${ }^{9}$ and locally administered lactobacilli prevent recurrences of urinary tract infections. ${ }^{10}$ To evaluate whether these products given orally are effective in preventing symptomatic recurrences of urinary tract infection we conducted an open, randomised, controlled trial.

\section{Participants and methods}

Study population and design

We recruited women during 1993-7 from the Finnish student health service at the University of Oulu and the occupational health centre for the staff of Oulu University Hospital. Women who had a urinary tract infection caused by Escherichia coli ( $\geqslant 10^{5}$ colony forming units $/ \mathrm{ml}$ in clean voided midstream urine) and were not taking antimicrobial prophylaxis were invited to participate. After giving informed consent, they were randomly allocated into three groups by using tables of random numbers and a block technique with a block size of six (fig 1).

The first group received $50 \mathrm{ml}$ of cranberrylingonberry juice concentrate (Maija, Marli, Finland) a day for six months, the second group received $100 \mathrm{ml}$ of Lactobacillus GG drink (Gefilus, Valio, Finland) five days a week for one year, and the third group served as an open control group. The cranberry-lingonberry juice contained $7.5 \mathrm{~g}$ cranberry concentrate and $1.7 \mathrm{~g}$ lingonberry concentrate in $50 \mathrm{ml}$ of water with no added sugars, and the lactobacillus drink contained $4 \times 10^{10} \mathrm{cfu}$ of Lactobacillus GG/100 ml. Participants were advised to prepare a drinkable juice by adding $200 \mathrm{ml}$ of water but no sweeteners to the $50 \mathrm{ml}$ of concentrate. Both drinks were commercially available at the time. The dosing frequencies and the duration of the prophylaxis were based on the availability of the products from our suppliers.

The first urinary tract infection (index) was treated with standard antimicrobials. Women had to provide a
Department of
Pediatrics,
University of Oulu,
Oulu, Fin-90220,
Finland 


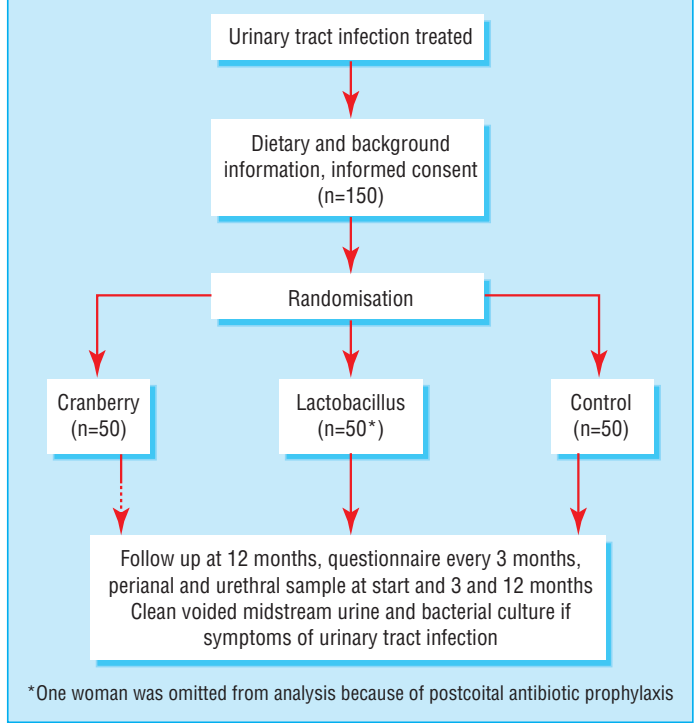

Fig 1 Design of trial

urine sample showing no bacterial growth three or more days after treatment before they were scheduled for follow up (fig 1). Each woman completed a questionnaire providing demographic data, medical history, and nutritional status at entry and a modified questionnaire at three month intervals. Women recorded use of the products on a self report sheet.

Whenever a participant had symptoms suggesting urinary tract infection (frequency, urgency, dysuria, haematuria, nocturia, fever, or back or flank pain), we obtained a clean voided midstream urine sample for culture. All the analyses were performed at the laboratory of clinical microbiology in Oulu University Hospital. The laboratory staff were unaware as to which of the treatment groups participants belonged. The samples were cultured immediately by standard procedures, and we used $\geqslant 10^{5} \mathrm{cfu} / \mathrm{ml}$ as the criterion for infection. If the participant reported a urinary tract infection that was not confirmed in our laboratory, we telephoned the diagnosing health care facility to obtain information on any culture obtained. Only cultures with $\geqslant 10^{5} \mathrm{cfu} / \mathrm{ml}$ were accepted and recorded as events. A urine sample with no bacterial growth was required between two episodes before they were regarded as separate events. Women who had three or more episodes in six months were offered antimicrobial prophylaxis.

We followed perianal and urethral colonisation with lactobacilli by taking swab samples at the start and at three and 12 months. The protocol was evaluated and approved by the ethics committee of the medical faculty of the University of Oulu.

\section{Sample size}

The study end point was the first recurrence of urinary tract infection. We calculated the sample size based on the assumption that at least $30 \%$ of women will experience a recurrence within a year. ${ }^{3}$ We considered a reduction in recurrences to $10 \%$ as clinically important. To detect such a reduction with a two tailed $\alpha$ of 0.05 and a power of $80 \%$, we needed 70 women in each group.

Recruitment had to be stopped prematurely because the cranberry juice supplier stopped produc- ing the juice. A total of 150 women gave their informed consent and were randomly allocated into three groups, 50 in each. One subject in the lactobacillus group who was taking postcoital antimicrobials was excluded from the analysis.

\section{Statistical methods}

We used the Kaplan-Meier method to analyse the cumulative first recurrence and the Breslow test to assess the differences in occurrences at the end of the study. We calculated the incidence density by adding the total number of episodes of urinary tract infection and the time at risk in each group and then calculating the rate of episodes per person year at risk. Each woman contributed days at risk until she dropped out, became pregnant, started antimicrobial prophylaxis, or the follow up ended. The differences in incidence of urinary tract infection between the groups were tested assuming that the occurrence of infection follows Poisson's distribution. We used Cox regression analysis to control for the baseline risk factors for infection.

\section{Results}

The groups were similar in their baseline characteristics with regard to the risk of urinary tract infection (table 1). The subjects had previously consumed the vaccinium berry or lactobacillus products only occasionally. Only 13 women dropped out of the study: four $(8 \%)$ in the cranberry group, four $(8 \%)$ in the lactobacillus group, and five $(10 \%)$ in the control group, usually because of moving away. There were no major changes in nutritional status during the follow up apart from the interventions.

During the six months, eight $(16 \%)$ women in the cranberry group, $19(39 \%)$ in the lactobacillus group, and $18(36 \%)$ in the control group had at least one episode of urinary tract infection. This is a $20 \%$ reduction in absolute risk in the cranberry group compared with the control group (95\% confidence interval 3\% to $36 \%$, $\mathrm{P}=0.023$, number needed to treat $=5,95 \%$ confidence interval 3 to 34$)$. The numbers who had had a recurrence at 12 months were 12,21 , and 19 in the cranberry, lactobacillus, and control groups respec-

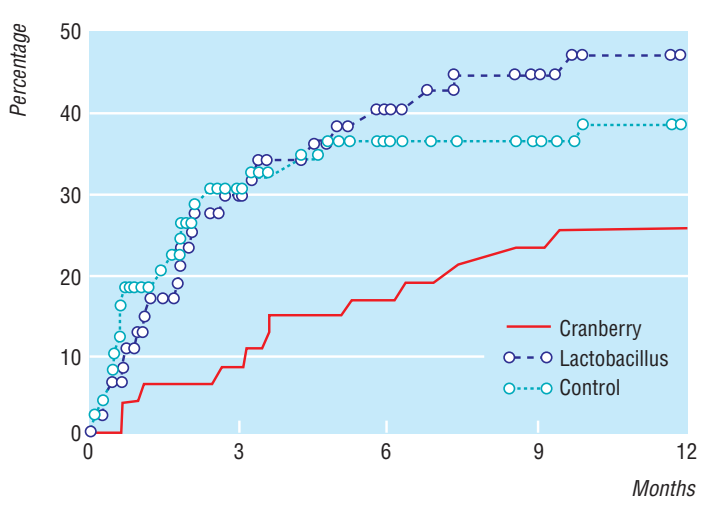

Fig 2 Cumulative rate of first recurrence of urinary tract infection during 12 month follow up in women receiving cranberry juice for six months, Lactobacillus GG drink for 12 months, or no intervention. The difference between groups was significant $(P=0.023$ at 6 months, 0.048 at 12 months). Occurrence of urinary tract infection was significantly lower in cranberry group than in control group ( $\mathrm{P}=0.014$ at 6 months, 0.052 at 12 months) 
tively. The cumulative first recurrence of urinary tract infection differed significantly between the groups throughout the trial ( $\mathrm{P}=0.048$ at 12 months; fig 2). Recurrence during the study period was significantly lower in the cranberry group than in the control group ( $\mathrm{P}=0.014$ at 6 months, $\mathrm{P}=0.052$ at 12 months).

We also did an analysis based on the assumption that women who dropped out of the intervention groups subsequently had a urinary tract infection whereas those who left the control group did not, but the differences in the occurrence of the first urinary tract infection remained significant $(\mathrm{P}=0.046$ at 12 months). After we standardised for age, history of urinary tract infection, antimicrobial use, intercourse frequency, and fluid intake at the start of the study, the only explaining variable for the prevention of recurrences was cranberry juice $(\mathrm{P}=0.019$, Cox regression analysis).

There were 98 episodes of urinary tract infection altogether during follow up, of which 21 (21\%) occurred in the cranberry group, $39(40 \%)$ in the lactobacillus group, and $38(39 \%)$ in the control group. The difference of 0.36 in incidence densities between the cranberry and control group was significant (table 2). One woman in the cranberry group had to start antimicrobial prophylaxis, compared with five and three in the lactobacillus and control groups, respectively.

The causative bacterium was $E$ coli in $80 \%$ of the episodes (table 2). The proportion of episodes caused by $E$ coli did not differ between the study groups. The number of women with perianal or urethral cultures positive for lactobacilli at any time during follow up was similar in all three groups; there were $32(71 \%)$ in the cranberry group, $33(67 \%)$ in the lactobacillus group, and $37(82 \%)$ in the control group.

Fifty three women (54\%) returned information about consumption of the products. They had taken $91 \%$ of the prescribed cranberry doses and $88 \%$ of lactobacillus doses. There were no differences in the main results of the stratified analysis between those giving this information or not (data not shown). No adverse events were reported except occasional complaints about the bitter taste of the cranberry juice.

\section{Discussion}

Our study confirms the common belief that symptomatic recurrences of urinary tract infection can be prevented with cranberry juice. In a recent review, the previous evidence for this was found inconclusive, ${ }^{11}$ although earlier reports had suggested a beneficial effect. ${ }^{12}$ The daily consumption of $300 \mathrm{ml}$ of cranberry juice reduced bacteriuria in postmenopausal women, but no beneficial effect was found among children with neurogenic bladder. ${ }^{913}$ Our study population represented a typical group of women at risk of recurrences of urinary tract infection. Such women are also the most willing and able to take preventive measures. We thus believe that our result is of both medical and practical importance.

In the United States, over 11 million women each year receive antimicrobials for urinary tract infection, costing over $\$ 1.6 \mathrm{bn}(£ 1100 \mathrm{~m}) .{ }^{1}$ Antimicrobial treatment and prophylaxis has resulted in increasing resistance to antimicrobials among uropathogenic bacteria. ${ }^{4}$
Table 1 Baseline characteristics of the subjects in the cranberry-lingonberry juice, Lactobacillus GG drink, and control groups. Values are numbers (percentages) of participants unless stated otherwise with percentages calculated from the number of women giving information on the question concerned

\begin{tabular}{|c|c|c|c|}
\hline Characteristics & $\begin{array}{c}\text { Cranberry } \\
(n=50)\end{array}$ & $\begin{array}{l}\text { Lactobacillus } \\
(\mathrm{n}=49)\end{array}$ & $\begin{array}{l}\text { Control } \\
(n=50)\end{array}$ \\
\hline \multicolumn{4}{|l|}{ Age (years): } \\
\hline Mean (SD) age & $32(9.8)$ & $30(11.8)$ & $29(10.5)$ \\
\hline Aged $<30$ & $30(60)$ & $36(73)$ & $36(72)$ \\
\hline Aged $30-55$ & $18(36)$ & $11(22)$ & $13(26)$ \\
\hline Aged $>55$ & $2(4)$ & $2(4)$ & $1(2)$ \\
\hline \multicolumn{4}{|l|}{ Intercourse frequency before intervention: } \\
\hline Less than once a week & $16(33)$ & $25(51)$ & $13(27)$ \\
\hline 1-2 times/week & $17(35)$ & $11(22)$ & $18(38)$ \\
\hline$>3$ times/week & $16(33)$ & $13(27)$ & $17(35)$ \\
\hline \multicolumn{4}{|l|}{ Intercourse frequency during follow up: } \\
\hline Less than once a week & $10(23)$ & $9(19)$ & $7(15)$ \\
\hline 1-2 times/week & $19(43)$ & $23(49)$ & $20(43)$ \\
\hline$>3$ times/week & $15(34)$ & $15(32)$ & $20(43)$ \\
\hline Used birth control during follow up* & $39(81)$ & $39(81)$ & $38(83)$ \\
\hline Pregnancy during follow up & $1(2)$ & $2(4)$ & $2(4)$ \\
\hline Mean No of antibiotic courses in past year & 2.6 & 2.1 & 2.1 \\
\hline Antimicrobials for urinary tract infection in past year & $44(90)$ & $41(85)$ & $41(85)$ \\
\hline \multicolumn{4}{|l|}{ Previous urinary tract morbidity: } \\
\hline Mean (range) No of infections & $6(1-30)$ & $6(1-30)$ & $6(1-20)$ \\
\hline Urinary tract anomalies $\dagger$ & $3(7)$ & $2(4)$ & 0 \\
\hline \multicolumn{4}{|l|}{ Antimicrobial prophylaxis for urinary tract infection: } \\
\hline Before intervention & $10(21)$ & $8(17)$ & $5(11)$ \\
\hline During follow up & $1(2)$ & $5(10)$ & $3(6)$ \\
\hline \multicolumn{4}{|l|}{ Mean (SD) daily fluid intake (I): } \\
\hline At start & $1.6(0.5)$ & $1.6(0.6)$ & $1.5(0.5)$ \\
\hline At 12 months & $1.6(0.7)$ & $1.7(0.7)$ & $1.6(0.5)$ \\
\hline
\end{tabular}

*Includes condom, coil, sterilisation, and oral contraception; no woman was using a diaphragm or spermicides.

†Includes grade II vesicourethral reflux (2), ureterocele (1), operated urethral malformation (1), and urinary incontinence (1).

Table 2 Incidence density of urinary tract infection per person year at risk and bacterial strains causing infection during 12 month follow up

\begin{tabular}{lccc} 
Urinary tract infection & Cranberry & Lactobacillus & Control \\
\hline Incidence density & $0.45^{\star}$ & 0.91 & 0.81 \\
\hline No $(\%)$ caused by E colit & $15(71)$ & $31(79)$ & $32(84)$ \\
\hline No $(\%)$ caused by other bacteria $\dagger$ & $6(29)$ & $8(21)$ & $6(16)$
\end{tabular}

*Difference between the cranberry and control groups ( 0.36 episodes/person year) was significant $(95 \%$ confidence interval 0.03 to $0.68, P=0.03$ ).

†Differences between the intervention groups and the control group were not significant $(P=0.24-0.59)$.

Cranberry juice provides an alternative tool for prevention of urinary tract infection that could result in decreased use of antimicrobials.

\section{Action of cranberry juice}

The berries of Vaccinium species such as cranberries and blueberries contain condensed tannins called proanthocyanidins. These can prevent the expression of the $\mathrm{P}$ fimbriae of $E \mathrm{coli}^{7}$ Proanthocyanidins are stable phenolic compounds that are widely distributed in nature, and some of them possess antiviral, antibacterial, antiadhesive, or antioxidant properties. ${ }^{14}{ }^{15}$ By inhibiting bacterial cell wall synthesis and cellular expression of adhesion molecules they inhibit bacterial adhesion to cellular surfaces. ${ }^{14-16}$ Tannin profiles differ between berries, and proanthocyanidin molecules of cranberry extracts consist mainly of epicatechin. ${ }^{17}$ The commercial product used here consisted mostly of cranberries ( $V$ oxycoccos) but also lingonberries ( $V$ vitisidaea), which to our knowledge have not been analysed for proanthocyanidin concentrations. 
What is already known on this topic

Up to $60 \%$ of women will have a urinary tract infection and a third of them will have several recurrences

Vaccinium berries and products containing lactobacilli may affect the coliform bacteria that cause urinary tract infection

\section{What this study adds}

$50 \mathrm{ml}$ of cranberry-lingonberry juice concentrate daily reduced recurrences of symptomatic urinary tract infection by about half compared with the control group

Lactobacillus GG drink had no effect on recurrence

Self treatment with cranberry juice may reduce the need for antimicrobials for recurrent urinary tract infection

$E$ coli is the most common uropathogenic bacterium, and its $\mathrm{P}$ fimbriae are thought to be the most important virulence factor in causing urinary tract infection. The blocking of fimbrial adhesion by cranberry juice prevents $E$ coli and other gram negative bacteria from colonising the uroepithelial cells. ${ }^{18-20}$ The juice may help to prevent urinary tract infection either by selecting less adhesive bacterial strains in the stool or by directly preventing $E$ coli from adhering to uroepithelial cells, or by both of these mechanisms. ${ }^{21} 22$ Our finding of no increase in recurrences after stopping cranberry prophylaxis supports the theory of bacterial selection in the stool.

\section{Lactobacillus}

The lactobacillus drink had no effect on urinary tract infection, possibly because we were unable to induce lactobacilli colonisation of the periurethral area. Lactobacilli have been shown to colonise the human intestine at doses of $10^{8-10} \mathrm{cfu} /$ day and to replace other bacterial species, especially coliform bacteria. ${ }^{83}$ Consumption five times a week may have been too infrequent. Intravaginal administration of lactobacilli has been found to reduce the number of coliform bacteria in the periurethral area and the number of urinary tract infections after antimicrobial treatment. ${ }^{10}$ In postmenopausal women, vaginal lactobacilli are replaced by enterobacteriaceae, increasing the risk of bacteriuria. This process can be reversed by intravaginal administration of oestriol. ${ }^{24}$

\section{Validity}

We had to stop our trial prematurely because the manufacturer of the cranberry juice stopped producing it. However, the difference in the occurrence of urinary tract infection between the cranberry and control group was clear and constant even in this limited sample size. The compliance follow up sheet was returned by only half of the subjects, but the reports suggested that the compliance was good and the stratified analysis showed no differences between the subjects who did and did not return the follow up sheet.

In conclusion, the daily consumption of $50 \mathrm{ml}$ of cranberry-lingonberry concentrate prevented recur- rences of symptomatic urinary tract infection in women, whereas the lactobacillus drink was ineffective. Cranberry juice reduced recurrences by about half. Since it is a natural food product and readily available, it seems a useful means for self administered prevention of urinary tract infection.

We thank Marli and Valio for providing the study products and Eeva-Liisa Lesonen and Tuulikki Ryhänen for recruiting women to the trial at the staff health centre.

Contributors: MU initiated and coordinated the formulation of the primary study hypothesis. TK participated in the formulation of study hypothesis and design and was responsible for coordinating the collection and entry of data. KS was responsible for the recruitment of the study subjects in the Finnish student health service. MU, MN, KS, and TK discussed core ideas, designed the study protocol, and participated in the data analysis, interpretation of the results, and writing the paper. TP was responsible for the statistical analysis, participated in the interpretation of the results, and contributed to the design and writing of the paper. MK was responsible for the quality of the laboratory analysis and contributed to the design and the writing of the paper. MU and TK are guarantors of the content of the paper.

Funding: Emil Aaltonen, Juho Vainio, and Alma and K A Snellman Foundations.

Competing interests: None declared.

1 Foxman B, Barlow R, D'Arcy H, Gillespie B, Sobel JD. Urinary tract infection: self-reported incidence and associated costs. Ann Epidemiol 2000; $10: 509-15$

2 Remis RS, Gurwith MJ, Gurwith D, Hargrett-Bean NT, Layde PM. Risk factors for urinary tract infection. Am J Epidemiol 1987;126:685-94.

3 Ikäheimo R, Siitonen A, Heiskanen T, Kärkkäinen U, Kuosmanen P, Lipponen $P$, et al. Recurrence of urinary tract infection in a primary care setting: analysis of a 1-year follow-up of 179 women. Clin Infect Dis 1996;22:91-9.

4 Gupta K, Scholes D, Stamm WE. Increasing prevalence of antimicrobial resistance among uropathogens causing acute uncomplicated cystitis in women. JAMA 1999;281:736-8.

5 Reid G. Probiotic therapy and functional foods for prevention of urinary tract infections: state of the art and science. Curr Infect Dis Rep 2000;2:518-22.

6 Gibson GR. Dietary modulation of the human gut microflora using prebiotics. BrJ Nutr 1998;80:209-12.

7 Howell AB, Vorsa N, Der Marderosian A, Foo LY. Inhibition of the adherence of P-fimbriated Escherichia coli to uroepithelial-cell surfaces by proanthocyanidin extracts from cranberries. N Engl J Med 1998;339: 1085-6.

8 Chan RC, Reid G, Irvin RT, Bruce AW, Costerton JW. Competitive exclusion of uropathogens from human uroepithelial cells by Lactobacillus whole cells and cell wall fragments. Infect Immun 1985;47:84-9.

9 Avorn J, Monane M, Gurwitz JH, Glynn RJ, Choodnovskiy I, Lipsitz LA. Reduction of bacteriuria and pyuria after ingestion of cranberry juice. JAMA 1994;271:751-4.

10 Reid G, Bruce AW, Taylor M. Influence of three-day antimicrobial therapy and lactobacillus vaginal suppositories on recurrence of urinary tract infections. Clin Ther 1992;14:11-6.

11 Jepson RG, Mihaljevic L, Craig J. Cranberries for preventing urinary tract infections. Cochrane Database Syst Rev 2000;2:CD001321.

12 Blatherwick NR. The specific role of foods in relation to the composition of the urine. Arch Intern Med 1914;14:409-50.

13 Schlager TA, Anderson S, Trudell J, Hendley JO. Effect of cranberry juice on bacteriuria in children with neurogenic bladder receiving intermittent catheterization.J Pediatr 1999;135:698-702.

14 Scalbert A. Antimicrobial properties of tannins. Phytochemistry 1991:30:3875-83

15 Sen CK, Bagchi D. Regulation of inducible adhesion molecule expression in human endothelial cells by grape seed proanthocyanidin extract. Mol Cell Biochem 2001;216:1-7.

16 Jones GA, McAllister TA, Muir AD, Cheng KJ. Effect of sainfoin (Onobrychis viciifolia Scop) condensed tannins on growth and proteolysis by four strains of ruminal bacteria. Appl Environ Microbiol 1994;60:1374-8.

17 Foo LY, Lu Y, Howell AB, Vorsa N. The structure of cranberry proanthocyanidins which inhibit adherence of uropathogenic P-fimbriated Escherichia coli in vitro. Phytochemistry 2000;54:173-81.

18 Schmidt DR, Sobota AE. An examination of the anti-adherence activity of cranberry juice on urinary and non-urinary bacterial isolates. Microbios 1988;55:173-81.

19 Zafriri D, Ofek I, Adar R, Pocino M, Sharon N. Inhibitory activity of cranberry juice on adherence of type 1 and type $P$ fimbriated Escherichia coli to eucaryotic cells. Antimicrob Agents Chemother 1989;33:92-8.

20 Ahuja S, Kaack B, Roberts J. Loss of fimbrial adhesion with the addition of Vaccinium macrocarpon to the growth medium of P-fimbriated Escherichia coli. J Urol 1998;159:559-62.

21 Sobota AE. Inhibition of bacterial adherence by cranberry juice: potential use for the treatment of urinary tract infections. J Urol 1984;131:1013-6. 
22 Ofek I, Goldhar J, Zafriri D, Lis H, Adar R, Sharon N. Anti-Escherichia coli adhesin activity of cranberry and blueberry juices. $N$ Engl J Med 1991;324:1599.

23 Saxelin M, Pessi T, Salminen S. Fecal recovery following oral administration of Lactobacillus strain GG (ATCC 53103) in gelatine capsules to healthy volunteers. Int J Food Microbiol 1995;25:199-203.
24 Raz R, Stamm WE. A controlled trial of intravaginal estriol in postmenopausal women with recurrent urinary tract infections. $N$ Engl J Med 1993;329:753-6.

(Accepted 23 March 2001) 